\title{
Generation of transgenic rice expressing heat shock protein genes under cool conditions
}

\author{
Hiroshi Yasuda*, Yoshiyuki Sagehashi, Etsuo Shimosaka, Yutaka Sato \\ Crop Breeding Research Division, NARO Hokkaido Agricultural Research Center, Hitsujigaoka 1, Sapporo, Hokkaido \\ 062-8555, Japan \\ *E-mail: hyasuda@affrc.go.jp Tel: +81-11-857-9478 Fax:+81-11-859-2178
}

Received June 26, 2013; accepted September 3, 2013 (Edited by K. Kato)

\begin{abstract}
The heat shock response of rice, including expression of heat shock transcription factors ( $H s f s)$, was investigated to elucidate the molecular regulation of its high-temperature tolerance. In silico analysis revealed that the rice genome encodes more than 19 species of $H s f$ genes that can be organized into three classes, A, B, and C. Rice seedlings treated with high temperature, express three class A $H s f$ genes ( $H s f A 2 a, H s f A 2 c$, and $H s f A 2 d)$ and two class B $H s f$ genes $(H s f B 2 b$ and $H s f B 2 c$ ) at significantly elevated levels. Transgenic rice plants overexpressing these three class A $H s f$ genes controlled by the rice actin 1 promoter or the wheat cold response (WCR) promoter expressed the transgenes, but did not express heat shock response genes such as small HSPs, whose expression is controlled by Hsfs. Treatment with geldanamycin, an inhibitor of HSP90, elevated the expression of HSPs in the WCR::Hsf transformant. Therefore, transgenic rice co-expressing WCR::Hsfs and a dominant negative HSP90 mutant were generated in which the heat shock response could be induced under WCR promoter-activating conditions. Successful induction of the heat shock response under cool conditions in this co-expression line suggests that HSP90 controls the heat shock response via the activity of $H s f$ in rice cells.
\end{abstract}

Key words: Heat shock factor, heat shock response, HSP90, transgenic rice.

Rice is a staple food in East Asia and comprises a large part of the daily diet of half of the people on earth. Therefore, stable rice yields are very important. Towards this goal, crops have been selected for environmental stress tolerance, including tolerance to extreme temperature, desiccation or salt tolerance. In fact, the rice-growing region has been spreading northward in Asia due both to global warming and the enhancement of cold tolerance through selective breeding. It will continue to be very important to confer stress tolerance to rice due to the broadening of growing regions and to enhance rice yields. Plants have developed mechanisms for dealing with environmental stress because they cannot physically avoid suboptimal environments, such as those with extreme temperatures, aridity, flooding, or chemical toxicity such as that in high salinity regions, or move to more optimal environments. To date, numerous experiment have been conducted to elucidate these stress tolerance mechanisms, and some transgenic plants that are highly tolerant to stresses such as low temperature, drought, or salt have been developed (Gao et al. 2011; Li et al. 2011; Xu et al. 2011).

Heat shock transcription factors $(H s f s)$ play a central role in the heat shock response. In fact, there are several reports describing high temperature tolerance conferred by $H s f s$ (Lee et al. 1995; Liu et al. 2009; Lohmann et al. 2004; Mishra et al. 2002; Ogawa et al. 2007; Prändl et al. 1998; Yokotani et al. 2007). Higher plants contain coding sequences for many more $H s f s$ in their genomes than do animals or Chlamydomonas. Mammals have three Hsf genes, whereas Chlamydomonas has only two Hsf genes, and only one of these (Hsf1) acts in the thermoresistance response (Morimoto 1998; Schulz-Raffelt et al. 2007). Yeast and Drosophila have only a single Hsf each; however, Arabidopsis, rice, and tobacco have at least 21, 25 or 26, and $17 \mathrm{Hsf}$ genes, respectively (Baniwal et al. 2004; Guo et al. 2008; Mishra et al. 2002; Mittal et al. 2009; von Koskull-Döring 2007). This high number of $H s f$ genes in higher plants suggests that plants have a complex and highly regulated system by which they cope with high temperatures and survive in a broader temperature range than animals or Chlamydomonas.

To assess the functions of individual $H s f s$, several transgenic approaches have been undertaken. Transgenic Arabidopsis expressing AtHsf1 fused to GUS under the control of the CaMV $35 S$ promoter constitutively expressed HSP 18 and HSP70 at approximately $20 \%$ of the maximum heat-inducible levels, and the AtHsf1

Abbreviations: HSP, heat shock protein; HSF, heat shock transcription factor; WCR, wheat cold response; GDM, geldanamycin. 
gene conferred basic thermotolerance via induction of heat-shock responsive genes (Lee et al. 1995). In contrast, overexpression of $H s f 3$, but not overexpression of Hsf4, in Arabidopsis derepressed the expression of several small HSPs (sHSPs) and led to an elevated basal thermotolerance (Prändl et al. 1998). However, there have been no prior reports analyzing the effects of overexpressing $H s f s$ in transgenic rice.

Rice is well known to be relatively tolerant to high temperatures because it originated in a tropical region; however, rice seedlings can also exhibit greater cold tolerance following a transient exposure to high temperature (Sato et al. 2001). This observation suggests that the heat-shock inducible genes also function to help plants endure cold temperatures. Recently, overexpression of Arabidopsis AtHsfA2 or rice OsHsfA2e conferred not only increased thermotolerance, but also increased salt-stress tolerance (Ogawa et al. 2007; Yokotani et al. 2007). Because the heat shock response confers tolerance not only to high temperature stress but also to other abiotic stresses such as low temperature or salt stress due to crosstalk among stress signaling pathways, elucidating the mechanism for high temperature response in rice will facilitate the development of crops tolerant to multiple abiotic stresses.

To investigate the induction mechanism for the heat shock response in rice, we generated transgenic rice overexpressing $H s f s$; however, these transformants did not induce the expression of $s H S P$ s, genes that are typically up-regulated during the heat shock response. When the seedlings of WCR::Hsf transformants were treated with $50 \mu \mathrm{M}$ geldanamycin (GDM), an inhibitor of HSP90, the expression levels of $s H S P$ s were higher than those of the non-transformant. These results strongly suggested that induction of the heat shock response in rice is controlled by HSP90 activity (Yamada et al. 2007). Therefore, we generated transgenic rice overexpressing $H s f s$ in a dominant negative HSP90 mutant. These transformants induced the expression of many $s H S P$ s under cool conditions that could activate the WCR (Wheat cold response) promoter. These results suggest that the heat shock response in rice is controlled by the activity of HSP90 and that the response in rice cells is more complicated than in Arabidopsis, a plant that originated in a temperate region. This is the first report of inducing the heat shock response under cool conditions.

\section{Materials and methods}

\section{Expression analysis of Hsfs and transgenes in rice seedlings by RT-PCR}

The full-length cDNA database at the Knowledge-Based Oryza Molecular Biological Encyclopedia (http://cdna01.dna.affrc. go.jp/cDNA/) was searched for full-length cDNA sequences of rice $H s f s$ and primer sets for each rice $H s f s$ were designed using
Primer Express software Version 3.0 (Applied Biosystems).

Sterilized rice seeds (Oryza sativa, cv. Oboroduki) were germinated and grown for 10 days on 1/2 MS (Murashige and Skoog Plant Salt Mixture, Nihon Pharmaceutical Co., Ltd., Tokyo, Japan) solid medium containing $0.3 \%$ Gelrite (Wako Pure Chemical Industries, Ltd., Osaka, Japan). The seedlings were transferred to an incubator at $42^{\circ} \mathrm{C}$ for $10 \mathrm{~min}, 60 \mathrm{~min}$, $120 \mathrm{~min}$, or $300 \mathrm{~min}$. The treated seedlings were homogenized under liquid nitrogen and RNA was extracted with RNAiso Plus (TAKARA, Kyoto, Japan). DNA in the samples was digested by DNaseI (Promega, Madison, WI) and then extracted with phenol:chloroform (1:1). Equal amounts of purified RNA were reverse-transcribed using a High Capacity cDNA Reverse Transcription kit (Applied Biosystems, Carlsbad, CA), and the products were used as templates for secondstrand synthesis, followed by quantitative PCR analysis. The quantitative PCR was performed with a 7300 Real Time PCR system (Applied Biosystems) using the Power SYBR Green PCR Master Mix (Applied Biosystems). Gene-specific primer sequences for ubiquitin, the $s H S P$ s, the Hsfs, and HSP90 (Table S1) were designed using Primer Express software Version 3.0. The primer set used to amplify each of the $s H S P$ s was designed in the highly conserved region of HSP16.9 (AK121025, AK242299), HSP17.4 (AK119717, AK073671, AK119239), and HSP18.0 (AK119664). Ubiquitin mRNA was used as an internal control in all experiments. Expression values are the averages of three independent experiments $\pm S D$.

\section{Overexpression of Hsf genes and mutated HSP9O in rice transformants}

Hsf genes (HsfA2a, AK069579; HsfA2c, AK072391; HsfA2d, AK066844) that are expressed in rice seedlings treated at high temperature were cloned and fused downstream of the rice actin 1 or WCR promoters (AB676782). The promoter::Hsf cDNAs with an added Nos terminator sequence were inserted between both border sequences of pBIN19 in which the antibiotic-resistance gene had been replaced with a hygromycin-resistance gene under control of the CaMV $35 \mathrm{~S}$ promoter. The double WCR::Hsf transformant was developed by crossing the WCR::HsfA2a and WCR::HsfA2c transformants. A dominant negative mutant of HSP90 (AK061896, a candidate for the Arabidopsis HSP90.2 homologue in rice (Yamada et al. 2007) was created in which $\mathrm{Asp}^{80}$ was converted to $\mathrm{Asn}^{80}$ by PCR (Pnaretou et al. 1998), and was designated the HSP90D80N gene. HSP90-D80N was fused downstream of the maize ubiquitin promoter, and the promoter::HSP90-D80N with an added Nos terminator were then inserted between both border sequences of the modified pBIN19. Transformation of Agrobacterium tumefaciens (strain EHA105) with the binary vector, inoculation of explants with the transformed Agrobacterium, and regeneration of transformed calli were conducted according to the method of Goto et al. (1999).

The WCR::HsfA2a/WCR::HsfA2c transformant was generated by crossing the WCR::HsfA2a transformant with the WCR::HsfA2a transformant. The WCR::HsfA2a/WCR:: 

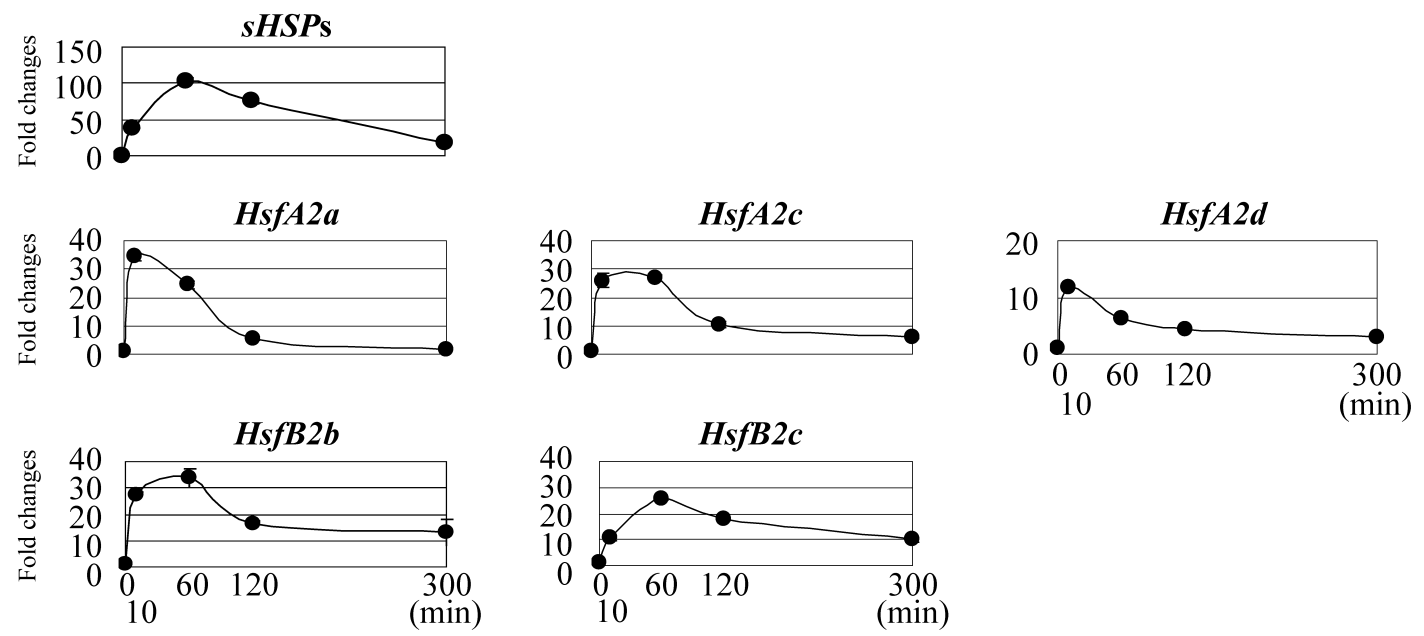

Figure 1. Expression levels of rice Hsfs under high temperature conditions. Total RNA was isolated from 10-day-old seedlings that were treated at $42^{\circ} \mathrm{C}$ for $10,60,120$, or $300 \mathrm{~min}$. The levels of each gene transcript were measured using quantitative RT-PCR. The values are normalized using ubiquitin mRNA as an internal control. The relative quantity of the untreated control was defined as 1 . Values are averages of three independent experiments $\pm S D$.

HsfA2c/HSP90-D80N transformant was generated by crossing the WCR::HsfA2a/WCR::HsfA2c transformant with the HSP90-D80N transformant.

\section{Treatment with geldanamycin}

Sterilized seeds of the WCR::Hsfs transformant were germinated and grown on $1 / 2 \mathrm{MS}$ solid medium containing $0.3 \%$ Gelrite at $27^{\circ} \mathrm{C}$. Ten-day-old seedlings were removed from the solid medium and treated with fresh $1 / 2$ MS liquid medium containing $50 \mu \mathrm{M}$ GDM (Tokyo Chemical Industry Co., Ltd., Japan) at $12^{\circ} \mathrm{C}$ overnight (ca. $16 \mathrm{~h}$ ) followed by incubation at $27^{\circ} \mathrm{C}$ for $30 \mathrm{~min}$. After this treatment, the expression level of transgenes and heat shock response genes were assessed according to the procedure as described above.

\section{Microarray analysis}

Microarray analysis was performed according to the manufacturer's instructions (Agilent Technologies, Palo Alto, CA). Total RNAs were prepared from leaves of wild type, HSP90-D80N, WCR::HsfA2a/WCR::HsfA2c, and WCR::HsfA2al WCR::HsfA2c/HSP90-D80N transformants (the transformants carrying the $W C R$ promoter were treated at $12^{\circ} \mathrm{C}$ overnight). The extracted RNAs were used to prepare reciprocally Cy3- or Cy5-labelled cRNA using the Quick Amp Labeling Kit (Agilent Technologies). Equal volumes of the two fluorescently labeled cRNAs were mixed and hybridized with the Agilent 44K Rice Oligo DNA Microarray RAP-DB (Agilent Technologies). The hybridized slides were scanned using a DNA microarray scanner (Agilent Technologies). Signal intensities were measured by feature extraction software (Agilent Technologies). The HSPs that are up-regulated more than 10-fold higher in the transformants than in the wild type are listed in Table S2-S5.

\section{Results}

\section{Expression profiles of rice Hsfs}

We searched for Hsf genes in the rice full-length cDNA database (Knowledge-Based Oryza Molecular Biological Encyclopedia) and found $19 H s f$ genes that are expressed in rice. When the expression of these $19 H s f$ genes was examined in high-temperature treated $\left(42^{\circ} \mathrm{C}\right)$ rice seedlings, only five $H s f$ genes (three genes from $H s f$ class A and two genes from class B) were highly induced (5fold higher expression than the control) by the hightemperature treatment (Figure 1). The expression levels of the three class A $H s f s$ ( $H s f A 2 a, H s f A 2 c$ and $H s f A 2 d)$ increased significantly within $10 \mathrm{~min}$; however, $H s f B 2 b$ and $H s f B 2 c$ expression levels increased continuously for up to $60 \mathrm{~min}$ after the high temperature treatment. The highest expression levels of four of the Hsfs (HsfA2a, $H s f A 2 c, H s f B 2 b$, and $H s f B 2 c$ ) were 25- to 35-fold higher than those of the non-treated control. Heat shock response genes such as $S H S P$ s (HSP16.9, HSP17.4 and HSP18.0) were also induced, followed by the expression of Hsf genes (Figure 1).

\section{Overexpression of HsfA2a, HsfA2c, and HsfA2d genes}

To further investigate the function and mechanism of the heat shock response, we generated transgenic plants that overexpress the $H s f A 2 a, H s f A 2 c$, and $H s f A 2 d$ genes under the control of the rice actin 1 promoter. The expression of each introduced gene was examined in 10-dayold seedlings of transformants in the T2 generation without heat shock treatment. The expression levels of $H s f A 2 c$ and HsfA2d in each act::Hsf transformant were approximately 10-fold and 6.2-fold higher than those of non-transformants, respectively (Table 1 ). The expression 
level of $H s f A 2 a$ in the act::HsfA2a transformant was only slightly higher (2.6-fold) than that of the nontransformants (Table 1). Further, the expression levels of $s H S P$ s in the act::Hsf transformants were also analyzed by quantitative PCR. The heat shock response genes were never induced in the seedlings of the act::Hsf transformants without prior heat shock treatment (Table $1)$.

Induction of heat shock response genes was not observed in any of the act::Hsf transformants. Next, we generated transformants expressing the HsfA2a, HsfA2c, and $H s f A 2 d$ genes under the control of a cold-inducible promoter originating from wheat $(W C R$, wheat cold response promoter). The WCR promoter is a stronger promoter than the rice actin 1 promoter when plants are treated with cold temperature (below $16^{\circ} \mathrm{C}$ ). In the $W C R:: H s f A 2 c$ transformants, the expression level of $H s f A 2 a$ and $H s f A 2 c$ were nearly 13 -fold and 180 -fold higher than that of the non-transformants, respectively, when seedlings of the transformant were treated at $12^{\circ} \mathrm{C}$ overnight (Table 1). In the WCR::HsfA2a and WCR::HsfA2d transformants, expression levels of HsfA2a and $H s f A 2 d$ were nearly 10 -fold and 20 -fold higher than those of the non-transformants, respectively (Table 1). The expression levels of $H s f A 2 c$ and HsfA2d in the transformants treated overnight at $12^{\circ} \mathrm{C}$ were higher than the expression levels in non-transformants treated at $42^{\circ} \mathrm{C}$ for $30-60 \mathrm{~min}$ (compare Figure 1 to Table 1 ).

We examined whether the heat shock response genes were induced in WCR::Hsf transformants that had been treated overnight at $12^{\circ} \mathrm{C}$ and then moved to $27^{\circ} \mathrm{C}$ for $30 \mathrm{~min}$ in order to maximize translation of $H s f$ genes and transcription of $s H S P$ s. Under these conditions, the expression of $s H S P$ s was barely induced, even when individual $H s f$ genes were highly expressed (Table 1).

We generated a double WCR::Hsf transformant by crossing WCR::HsfA2a with WCR::HsfA2c transformants since high temperature treatment of the wild type induced higher levels of $H s f A 2 a$ and $H s f A 2 c$ genes than that of the HsfA2d gene (Figure 1). When these transformants were treated overnight at $12^{\circ} \mathrm{C}$ (Table 2), the $H s f A 2 a$ and $H s f A 2 c$ genes were induced in each transformant. We examined whether the heat shock response was induced in the WCR::HsfA2a/WCR::HsfA2c double transformants treated under the same conditions and found that the heat shock response genes were slightly induced (Table 2). However, the induction level observed under cooler conditions was weaker than when the seedlings were treated at $42^{\circ} \mathrm{C}$ (Figure 1).

\section{Geldanamycin treatment}

Geldanamycin (GDM) is an antibiotic that induces the heat shock response in the absence of a heat shock treatment. GDM acts as an inhibitor of HSP90 that may inhibit the interaction between HSP90 and HSF (Yamada et al. 2007). To investigate the interaction between HSP90 and the introduced HSFs, we treated the WCR::Hsf transformants with GDM.

We assessed the expression level of $s H S P$ s in 10-dayold seedlings of WCR::Hsf transformants that were incubated in $1 / 2 \mathrm{MS}$ medium containing $50 \mu \mathrm{M}$ GDM with the cold treatment $\left(12^{\circ} \mathrm{C}\right.$ overnight $)$ to activate the $W C R$ promoter. The transcript levels of the HsfA2a, HsfA2c and HsfA2d genes in the GDM treated WCR::HsfA2a, WCR::HsfA2c and WCR::HsfA2d transformant were $20.52 \pm 7.6-, 33.33 \pm 3.79-$ and $13.71 \pm$

Table 1. Relative transcript abundance of $H s f$ genes and $s H S P s$ in the act::Hsfs and WCR::Hsfs transformants.

\begin{tabular}{lrrrc}
\hline & HsfA2a & HsfA2c & HsfA2d & sHSPs \\
\hline act::HsfA2a & $2.61 \pm 0.57$ & $0.89 \pm 0.16$ & $0.85 \pm 0.20$ & $1.39 \pm 0.35$ \\
act::HsfA2c & $1.51 \pm 0.30$ & $10.07 \pm 0.30$ & $1.03 \pm 0.08$ & $1.76 \pm 0.10$ \\
act::HsfA2d & $2.87 \pm 1.34$ & $1.86 \pm 1.35$ & $6.19 \pm 3.38$ & $1.82 \pm 0.98$ \\
WCR::HsfA2a & $9.78 \pm 7.45$ & $1.14 \pm 0.78$ & $0.71 \pm 0.35$ & $0.78 \pm 0.06$ \\
WCR::HsfA2c & $12.91 \pm 7.71$ & $183.8 \pm 67.7$ & $0.96 \pm 0.35$ & $2.20 \pm 0.54$ \\
WCR::HsfA2d & $3.42 \pm 0.63$ & $1.58 \pm 0.54$ & $21.93 \pm 3.22$ & $0.97 \pm 0.26$
\end{tabular}

Transcript abundance was normalized to the expression level of $u$ biquitin and compared with transcript abundance in the non-transformant from same treatment that was defined as 1 . Relative transcript abundance of $H s f A 2 a, H s f A 2 c$ and $H s f A 2 d$ in 10 days old seedlings of the WCR::Hsf transformants were measured after an overnight treatment at $12^{\circ} \mathrm{C}$. The relative transcript abundance of $s H S P$ s was measured after an overnight treatment at $12^{\circ} \mathrm{C}$ and then $27^{\circ} \mathrm{C}$ for 30 min. Values are averages of three independent experiments $\pm S D$.

Table 2. Relative transcript abundance of Hsf genes, HSP90 and sHSPs in the WCR::HsfA2a/WCR::HsfA2c and WCR::HsfA2a/WCR::HsfA2c/ HSP90-D80N transformants.

\begin{tabular}{|c|c|c|c|c|}
\hline & $H s f A 2 a$ & $H s f A 2 c$ & $\begin{array}{c}\text { HSP90 } \\
\text { (including HSP90-D80N) }\end{array}$ & sHSPs \\
\hline WCR::HsfA2a/WCR::HsfA2c & $5.64 \pm 3.60$ & $9.56 \pm 1.59$ & $0.80 \pm 0.27$ & $8.50 \pm 1.44$ \\
\hline WCR::HsfA2a/WCR::HsfA2c/HSP90-D80N line 9 & $9.36 \pm 1.45$ & $7.43 \pm 2.54$ & $1.94 \pm 0.54$ & $20.61 \pm 1.56$ \\
\hline
\end{tabular}

Transcript abundance was normalized to the expression level of ubiquitin and compared with transcript abundance in the non-transformant from same treatment that was defined as 1. Relative transcript abundance of HsfA2a, HsfA2c and HSP90-D80N (including HSP90-D80N) in 10 days old seedlings of the triple transformants was measured after an overnight treatment at $12^{\circ} \mathrm{C}$. Values are averages of three independent experiments $\pm S D$. 
3.93-fold higher than the same treatment of wild type, respectively.

In the GDM-treated WCR::HsfA2a, WCR::HsfA2c, and WCR::HsfA2d transformants incubated overnight at $12^{\circ} \mathrm{C}$, the expression of $s H S P$ s was induced to 8-, 9-, and 5 -fold higher levels, respectively, than that of the non-transformant (Figure 2). These results suggest that $H s f A 2 a$ and $H s f A 2 c$ are important for induction of $s H S P$ expression and that HSP90 is also a factor responsible for induction of $s H S P$ s.

\section{Co-expression of Hsf genes and HSP90-D80N}

The results of GDM treatment strongly suggested that the heat shock response in rice is controlled by the activity of HSP90. Therefore, we generated transgenic rice overexpressing the dominant negative mutant of HSF90 (HSP90-D80N). Some of the HSF90-D80N transformants in the T0 generation had dwarf phenotypes and were not selected for further investigation. None of the selected lines in the T2 generation had dwarf phenotypes or alterations in leaf number or seed fertility problems when

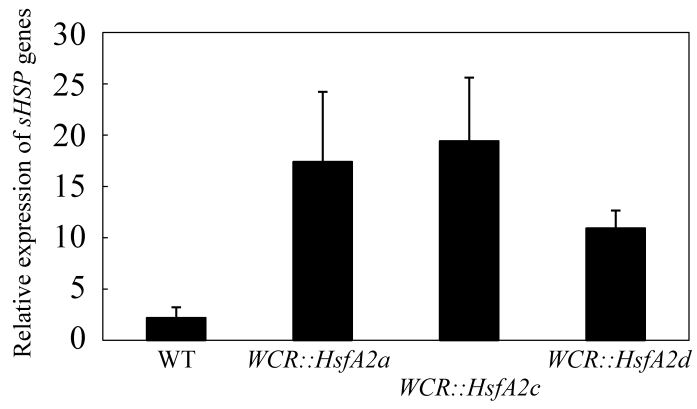

Figure 2. Expression levels of $s H S P s$ in GDM-treated WCR::Hsfs transformants. The 10-day-old seedlings were incubated with $50 \mu \mathrm{M}$ GDM according to the procedure outlined in Materials and methods. The values were normalized to the expression level of ubiquitin mRNA as a standard. The value for the non-transformant (WT) without GDM treatment was defined as 1 . Values are averages of three independent plants $\pm S D$. plants were grown in the glasshouse. The expression level of HSP9O in the HSP90-D80N transformant was $2.79 \pm 1.17$-fold higher than that of wild type. The HSP90-D80N transformant was crossed with the WCR::Hsf transformant to generate the WCR::HsfA2al WCR::HsfA2c/HSP90-D80N transformant, which was expected to induce the expression of $s H S P$ s after exposure to cool $\left(12^{\circ} \mathrm{C}\right)$ temperatures. The expression levels of HSP9O in the transformants and wild type were not elevated by the cool temperature treatment.

Microarray analysis was used to assess the expression of $s H S P$ s in the HSP90-D80N, WCR:: HsfA2a/WCR::HsfA2c, and WCR::HsfA2a/WCR::HsfA2cl HSP90-D80N transformants. We found that overexpression of HSP90-D80N alone did not induce the heat shock response in the transformant (Figure 3), as only one up-regulated gene (heat shock protein DnaJ family protein) with $>10$-fold elevated expression was detected (Table S2). On the other hand, the expression of seven $H S P$ s was induced 10-fold over that of wild type in the cool-temperature treated WCR::HsfA2a/WCR::HsfA2c transformant (Figure 3, Table S3).

When gene expression was compared between cool temperature-treated wild type and the WCR::HsfA2a/WCR::HsfA2c/HSP $90-D 80 \mathrm{~N}$ transformants, many HSP genes, including high molecular-weight HSPs, were induced in the transformants (Figure 3, Table S4, S5). The expression levels of HSPs in the WCR::HsfA2a/WCR::HsfA2c/ HSP90-D80N transformants were higher and the number of species of HSPs whose expression was induced was greater than in the WCR::HsfA2a/WCR::HsfA2c transformant. These results clearly suggested that the heat shock response could be induced by cool conditions in rice cells in this system (Figure 2, Table S4, S5) and that $H s f s$ under the control of HSP90 are necessary for the expression of the heat shock response in rice cells.
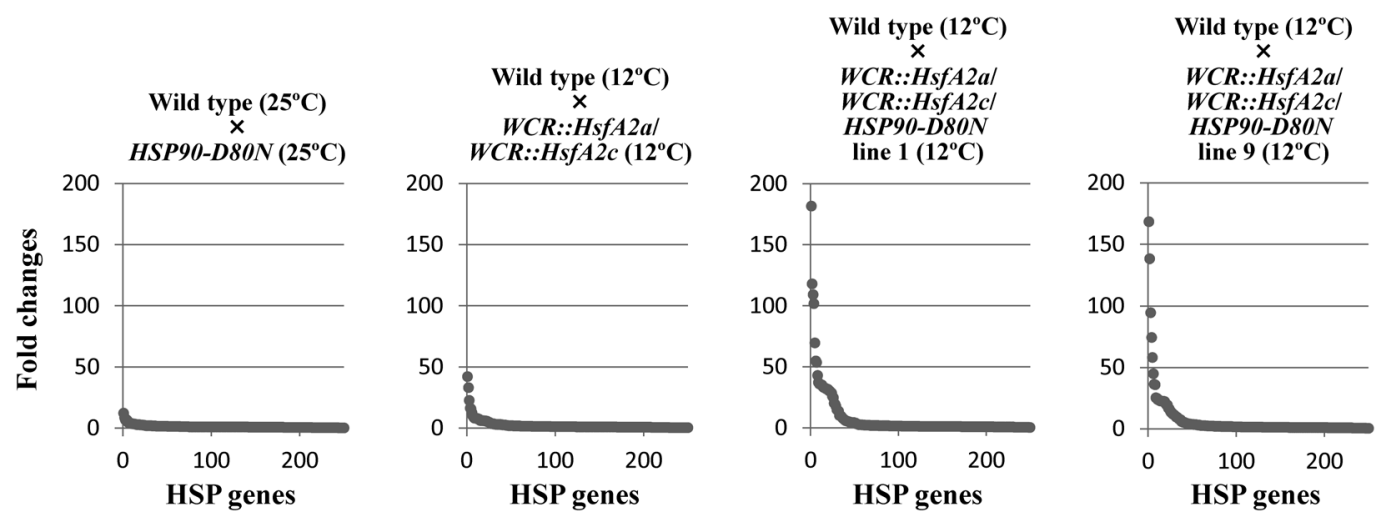

Figure 3. Expression levels of all HSPs on rice DNA oligo microarrays in normal $\left(25^{\circ} \mathrm{C}\right)$ and cool temperature $\left(12^{\circ} \mathrm{C}\right)$-treated transformants. The methods used for microarrays are described in Materials and methods. The value for the HSPs of wild type under same temperature condition was defined as 1 . The horizontal axis indicates the spots of HSPs on the array in descending order of fold change, and the combination of microarray experiments is described above the chart. 


\section{Discussion}

We detected at least $19 \mathrm{Hsfs}$ in the rice full-length cDNA database and found that these Hsfs could be divided into three classes, based on whether the HR-A/B region contained an insertion of either 21 (class A), 0 (class B), or 7 (class $\mathrm{C}$ ) amino acid residues between the $\mathrm{A}$ and $\mathrm{B}$ regions (Nover et al. 2001). In general, the rice genome encodes at least $26 \mathrm{Hsfs}$, including one variant that lacks a DNA-binding domain, a nuclear localization signal, a nuclear export signal, and an AHA-type activation domain (Guo et al. 2008). Twenty-two of these 26 $H s f s$ were induced throughout the rice plant by heat shock treatment. When 10-day-old seedlings were heat shocked, at least $16 \mathrm{Hsfs}$ were up-regulated by $>2$-fold (Mittal et al. 2009). In our study, the expression levels of only five Hsfs (HsfA2a, HsfA2c, HsfA2d, HsfB2b, and $H s f B 2 c$ ) increased more than 5 -fold in response to high temperature, and some other $H s f s$ such as $H s f A 2 b$, HsfA2e, HsfA4b, HsfA4d, HsfA7, HsfA9, HsfB $4 b$, and $H s f C 2 a$, were slightly up-regulated from 2- to 5 -fold in the seedlings treated after high temperature treatment (data not shown). Based on their high level of induction in response to high temperature, these five Hsfs (Figure 1) seem to be chiefly responsible for the expression of the heat shock response genes in rice seedlings.

$H s f A 2 a, H s f A 2 c$, and HsfA2d responded to high temperature rapidly, within $10 \mathrm{~min}$. Similar results were reported by Liu et al. (HsfA2c (Liu et al. 2005), HsfA2d (Liu et al. 2010)), and the expression of HsfA2c reached a maximum level within $5 \mathrm{~min}$ after high temperature treatment. For class B $H s f s, H s f B 2 b$ and $H s f B 2 c$, the level of expression continued to increase until $60 \mathrm{~min}$ after the high temperature treatment. These results suggest that $H s f A 2 a, H s f A 2 c$, and $H s f A 2 d$ play an important role in the initial reaction to the high temperature response, and that $H s f B 2 b$ and $H s f B 2 c$ play a role in prolonging or intensifying the response to the treatment.

In tomato, HsfAla (LeHsfAla) acts as the "master regulator" of the heat stress response (Mishra et al. 2002), whereas, in Arabidopsis, at least two or more $H s f A 1 s$ play the master regulator role despite similarities in the composition of the Hsf families of tomato and Arabidopsis (Liu et al. 2011; Nishizawa-Yokoi et al. 2011; Yoshida et al. 2011). To date, the master regulator of the heat stress response in rice has not been identified. The difficulty in identifying a master regulator may suggest that the heat stress responses in rice are more complex than that of tomato, in which the heat shock response is regulated by one gene (LeHsfA1a). Our results are good agreement with the suggestion that the expression of sHSPs were slightly induced in the double transformants containing WCR::HsfA2a and WCR::HsfA2c at the low temperature, although $s H S P$ s were not induced in the single transformants containing the WCR::HsfA2x gene under identical conditions (Table 2). These results also indicated that the mechanism for induction of the heat shock response in rice is similar to that in Arabidopsis, but the species of HsfA is different. To elucidate this complex mechanism in rice, we generate transformants that simultaneously overexpressed multiple $H s f s$.

The function of $H s f s$ as transcriptional activators is based on the structure of the proteins' four domains. Three domains (DNA-binding, oligomerization, and localization domains) are present in all three classes (A, B, and C) of Hsfs, but the activation domain only exists in class A Hsfs (Kotak et al. 2004; Nover et al. 2001). The HsfA1 and HsfA2 families are responsible for the heat shock response (Busch et al. 2005; Mishra et al. 2002; Nishizawa et al. 2006; Nishizawa-Yokoi et al. 2011; Ogawa et al. 2007; Schramm et al. 2006). Notably, rice has an HsfA2 group consisting of five genes (Baniwal et al. 2004; von Koskull-Döring et al. 2007). Some of the HsfA2 group genes reacted to heat shock treatment within 10 min (Figure 1), but HsfAla did not (data not shown). These results suggest that the activation of the heat shock response is regulated by the A2-type of $H s f s$ in rice cells. If the heat shock response is regulated by the A2-type of $H s f$ s, then HsfAla may be associated with another stage such as amplification or maintenance of the heat shock response. When OsHsfA2e was overexpressed in Arabidopsis, the expression of heat shock response genes (HSPs, Gols1, and others) was induced in the transformants in the absence of heat stress, and the transformants had an enhanced tolerance to high temperature (Yokotani et al. 2007). In contrast, when OsHSF7 (similar to HsfA2d) was overexpressed in Arabidopsis, the transformants did not induce the expression of most heat shock response genes under normal conditions, except for a few HSPs (Liu et al. 2009). These results suggested that OsHsfA2e may be one of the master regulators like LeHsfAla and that OsHsfA2e is necessary for activation of the heat shock response in Arabidopsis, unlike OsHSF7. In our experiments, transgenic rice overexpressing $H s f A 2 e$ under the control of the rice actin 1 and WCR promoters did not induce heat shock response genes under normal and cool temperature conditions, respectively (data not shown). Furthermore, we developed transgenic rice overexpressing A2-type Hsfs (HsfA2a, HsfA2c and $H s f A 2 d)$ that were inducible with high temperature treatment (Figure 1). Four (HsfA2a, HsfA2c, HsfA2d and $H s f A 2 e$ ) out of five class A Hsfs could not induce a heat shock response, unlike in Arabidopsis (Nishizawa et al. 2006; Ogawa et al. 2007; Prändl et al. 1998; Yokotani et al. 2007) and tomato (Mishra et al. 2002), even when these transcription factor genes were overexpressed in rice cells. These results suggest at least two possibilities: (i) the transcripts of introduced $H s f$ s were not translated due to an unknown mechanism in rice cells or (ii) the levels 
of transcripts produced by the transgene were too low to induce the heat shock response. However, the transcript levels of $H s f A 2 c$ and $H s f A 2 d$ in each transformant under control of the WCR promoter treated at $4^{\circ} \mathrm{C}$ were higher than the expression level in non-transformants treated at $42^{\circ} \mathrm{C}$ (Figure 1, Table I). Although translation product accumulation was not examined, the level of overexpression and efficient translation of $H s f A 2$ were very important for inducing the heat shock response (Ogawa et al. 2007). Therefore, we propose that rice cells may inhibit the translation of Hsf genes under normal temperatures.

To investigate further the induction machinery of the heat shock response in rice cells, the WCR::Hsf transformants were treated with GDM, an antibiotic that induces the heat shock response in Arabidopsis without heat shock treatment (Nishizawa-Yokoi et al. 2011; Yamada et al. 2007). After GDM treatment, the expression level of heat shock response genes (sHSPs) was enhanced in all rice transformants, even if the expression level was lower than when the seedlings were treated with high temperature (Figure 2). In addition, the expression level of HSP70 was also enhanced several-fold relative to that of the non-transformants upon the same treatment (data not shown). These results indicate that the inhibition of HSP90 activity may require the onset of a heat shock response. In this report, we found that the GDM-induced expression of heat shock response genes was enhanced by the pre-expression of HsfA2 genes, especially $H s f A 2 a$ and $H s f A 2 c$. This result suggests that the transgenic product of $H s f A 2$ was sequestered by endogenous HSP90; however, the transcript level of HSP90 was not enhanced in the WCR::Hsf transformants without GDM treatment (data not shown). These findings suggest the existence of other mechanism(s) in rice cells that inhibit the activity of $H s f s$ in all transformants.

To elucidate the relationship between the activity of HSP90 and the initiation of the heat shock response in rice cells, we are generating another rice transformant that expresses the dominant negative form of HSP90 (HSP90-D80N), and are further analyzing the relationship between the activity of HSP90 and the heat shock response. When expressing HSP90-D80N under control of the maize ubiquitin promoter, only one HSP (a DnaJ family protein) was induced in the transformant (Figure 3, Table S2). However, many other genes in the transformant were strongly up- or down-regulated compared to the wild type (data not shown). These results suggest that the overexpression of the mutant form of HSP90 affects the expression of many genes due to dysfunction of the chaperone activity of HSP90.

The WCR::HsfA2a/WCR::HsfA2c transformant was able to induce a small number of HSPs (seven spots on the array with greater than 10-fold elevated expression) under cool conditions (Table 2, S3). These results suggest that simultaneous expression of some of the class A Hsfs would induce the heat shock response in the transformants. However, the induction rate in multiple Hsf transformants was very low compared to that of the wild type treated at $42^{\circ} \mathrm{C}$ (Figure 1).

The transformants overexpressing HSP90-D80N, $H s f A 2 a$, and $H s f A 2 c$ induced the heat shock response under cool conditions (Figure 3, Table 2, S4, S5). In particular, the transformant designated as line 1 expressed heat shock protein genes to a significant degree (Table 2, S4). When the results of microarray analysis for line 1 were compared to those for line 9 transformants, the expression levels of HSPs were found to be elevated with increasing levels of transgene expression in line 1 (Table 2, S4, S5). These results indicate that if the transgenes (class A Hsfs and HSP90-D80N) could be expressed at higher levels, the heat shock response could be more strongly induced. Furthermore, when the heat shock response of line 9 was compared to that of the WCR::HsfA2a/WCR::HsfA2c transformant, we confirmed that the expression level of the $s H S P$ s was controlled by the expression of HSP90-D80N. These results strongly suggested that the activity of HSP90 was very important for the induction of the heat shock response in rice cells. In future studies, we will also assess the cold tolerance of the WCR::HsfA2a/WCR::HsfA2c/HSP90-D80N transformants.

To our knowledge, this is the first report of a rice transformant that can induce the heat shock response under cool conditions. These results provide a method to manipulate environmental stress tolerance in crops at the molecular level.

\section{Acknowledgements}

The authors thank Dr. Y. Nagamura and Ms. R. Motoyama for instructing us in methods for microarray analysis. We also thank Ms. R. Kihara for providing technical assistance.

\section{References}

Baniwal SK, Bharti K, Chan KY, Fauth M, Ganguli A, Kotak S, Mishra SK, Nover L, Port M, Scharf KD, et al. (2004) Heat stress response in plants: a complex game with chaperons and more than twenty heat stress transcription factors. J Biosci 29: 471-487

Busch W, Wunderlich M, Schöffl F (2005) Identification of novel heat shock factor-dependent genes and biochemical pathways in Arabidopsis thaliana. Plant J 41: 1-14

Gao T, Wu Y, Zhang Y, Liu L, Ning Y, Wang D, Tong H, Chen S, Chu C, Xie Q (2011) OsSDIR1 overexpression greatly improves drought tolerance in transgenic rice. Plant Mol Biol 76: 145-156

Goto F, Yoshihara T, Shigemoto N, Toki S, Takaiwa F (1999) Iron fortification of rice seed by the soybean ferritin gene. Nat Biotechnol 17: 282-286

Guo J, Wu J, Ji Q, Wang C, Luo L, Yuan Y, Wang Y, Wang J (2008) Genome-wide analysis of heat shock transcription factor families in rice and Arabidopsis. J Genet Genomics 35: 105-118 
von Koskull-Döring P, Scharf K-D, Nover L (2007) The diversity of plant heat stress transcription factors. Trends Plant Sci 12: 452-457

Kotak S, Port M, Ganguli A, Bicker F, von Koskull-Döring P (2004) Characterization of C-terminal domains of Arabidopsis heat stress transcription factors (Hsfs) and identification of a new signature combination of plant class A Hsfs with AHA and NES motifs essential for activator function and intracellular localization. Plant J 29: 98-112

Lee J, Hübel A, Schöffl F (1995) Derepression of the activity of genetically engineered heat shock factor causes constitutive synthesis of heat shock proteins and increased thermotolerance in transgenic Arabidopsis. Plant J 8: 603-612

Li HW, Zang BS, Deng XW, Wang XP (2011) Overexpression of the trehalose-6-phosphate synthase gene OsTSP1 enhances abiotic stress tolerance in rice. Planta 234: 1007-1018

Liu AL, Zou J, Zhang XW, Zhou XY, Wang WF, Xiong XY, Chen LY, Chen XB (2010) Expression profiles of class A rice heat shock transcription factor genes under abiotic stresses. J Plant Biol 53: 142-149

Liu HC, Liao HT, Charng YY (2011) The role of class A1 heat shock factors (HSFAla) in response to heat and other stresses in Arabidopsis. Plant Cell Environ 34: 738-751

Liu JG, Qin QL, Zhang Z, Peng RH, Xiong AS, Chen JM, Yao QH (2009) OsHSF7 gene in rice, Oryza sativa L., encodes a transcription factor that functions as a high temperature receptive and responsive factor. $B M B$ Rep 42: 16-21

Liu JG, Yao QH, Zhang Z, Peng RH, Xiong AS, Xu F, Zhu H (2005) Isolation and characterization of a cDNA encoding two novel heat-shock factor OsHSF6 and OsHSF12 in Oryza sativa L. J Biochem Mol Biol 38: 602-608

Lohmann C, Eggers-Schumacher G, Wunderlich M, Schöffl F (2004) Two different heat shock transcription factors regulate immediate early expression of stress genes in Arabidopsis. Mol Genet Genomics 271: 11-21

Mishra SK, Tripp J, Winkelhaus S, Tschiersch B, Theres K, Nover L, Scharf KD (2002) In the complex family of heat stress transcription factors, HsfAl has a unique role as master regulator of thermotolerance in tomato. Genes Dev 16: 1555-1567

Mittal D, Chakrabarti S, Sarkar A, Singh A, Grover A (2009) Heat shock factor gene family in rice: Genomic organization and transcript expression profiling in response to high temperature, low temperature and oxidative stresses. Plant Physiol Biochem 47: 785-795

Morimoto RI (1998) Regulation of the heat shock transcriptional response: cross talk between a family of heat shock factors, molecular chaperones, and negative regulators. Genes Dev 12: 3788-3796

Nishizawa A, Yabuta Y, Yoshida E, Maruta T, Yoshimura K, Shigeoka S (2006) Arabidopsis heat shock transcription factor A2 as a key regulator in response to several types of environmental stress. Plant J 48: 535-547

Nishizawa-Yokoi A, Nosaka R, Hayashi H, Tainaka H, Maruta T, Tamoi M, Ikeda M, Ohme-Takagi M, Yoshimura K, Yabuta Y, et al. (2011) HsfA1d and HsfAle involved in the transcriptional regulation of $H s f A 2$ function as key regulators for the Hsf signaling network in response to environmental stress. Plant Cell Physiol 52: 933-945

Nover L, Bharti K, Döring P, Mishra SK, Ganguli A, Scharf KD (2001) Arabidopsis and the heat stress transcription factor world: How many heat stress transcription factors do we need? Cell Stress Chaperones 6: 177-189

Ogawa D, Yamaguchi K, Nishiuchi T (2007) High-level overexpression of the Arabidopsis HsfA2 gene confers not only increased thermotolerance but also salt/osmotic stress tolerance and enhanced callus growth. J Exp Bot 58: 3373-3383

Pnaretou B, Prodromou C, Roe SM, O’Brien R, Ladbury JE, Piper PW, Pearl LH (1998) ATP binding and hydrolysis are essential to the function of the Hsp90 molecular chaperone in vivo. EMBO J 17: 4829-4836

Prändl R, Hinderhofer K, Eggers-Schumacher G, Schöffl F (1998) HSF3, a new heat shock factor from Arabidopsis thaliana, derepresses the heat shock response and confers thermotolerance when overexpressed in transgenic plants. Mol Gen Genet 258: 269-278

Sato Y, Murakami T, Funatsuki H, Matsuba S, Saruyama H, Tanida M (2001) Heat shock-mediated APX gene expression and protection against chilling injury in rice seedlings. J Exp Bot 354: 145-151

Schramm F, Ganguli A, Kiehlmann E, Englich G, Walsh D, von Koskull-Döring P (2006) The heat stress transcription factor HsfA2 serves as a regulatory amplifier of a subset of genes in the heat stress response in Arabidopsis. Plant Mol Biol 60: 759-772

Schulz-Raffelt M, Lodha M, Schroda M (2007) Heat shock factor 1 is a key regulator of the stress response in Chlamydomonas. Plant J 52: 286-295

Xu M, Li L, Fan Y, Wan J, Wang L (2011) ZmCBF3 overexpression improves tolerance to abiotic stress in transgenic rice (Oryza sativa) without yield penalty. Plant Cell Rep 301: 1949-1957

Yamada K, Fukao Y, Hayashi M, Fukazawa M, Suzuki I, Nishimura M (2007) Cytosolic HSP90 regulates the heat shock response that is responsible for heat acclimation in Arabidopsis thaliana. J Biol Chem 282: 37794-37804

Yokotani N, Ichikawa T, Kondou Y, Matsui M, Hirochika H, Iwabuchi M, Oda K (2007) Expression of rice heat stress transcription factor OsHsfA2e enhances tolerance to environmental stress in transgenic Arabidopsis. Planta 227: 957-967

Yoshida T, Ohama N, Nakajima J, Kidokoro S, Mizoi J, Nakashima K, Maruyama K, Kim JM, Seki M, Todaka D, et al. (2011) Arabidopsis HsfA1 transcription factors function as the main positive regulators in heat shock-responsive gene expression. $\mathrm{Mol}$ Genet Genomics 286: 321-332 